\title{
ON POINT SETS IN VECTOR SPACES OVER FINITE FIELDS THAT DETERMINE ONLY ACUTE ANGLE TRIANGLES
}

\author{
IGOR E. SHPARLINSKI
}

(Received 14 March 2009)

\begin{abstract}
For three points $\mathbf{u}, \mathbf{v}$ and $\mathbf{w}$ in the $n$-dimensional space $\mathbb{F}_{q}^{n}$ over the finite field $\mathbb{F}_{q}$ of $q$ elements we give a natural interpretation of an acute angle triangle defined by these points. We obtain an upper bound on the size of a set $\mathcal{Z}$ such that all triples of distinct points $\mathbf{u}, \mathbf{v}, \mathbf{w} \in \mathcal{Z}$ define acute angle triangles. A similar question in the real space $\mathcal{R}^{n}$ dates back to P. Erdôs and has been studied by several authors.
\end{abstract}

2000 Mathematics subject classification: primary 05B25; secondary 11T23, 52C10.

Keywords and phrases: acute angle triangle, finite field, character sum.

\section{Introduction}

Recent remarkable results of Bourgain, Katz and Tao [3] on the sum-product problem in finite fields have stimulated a series of studies of finite field analogues of classical combinatorial and discrete geometry problems; see [2, 4-6, 8-14, 17, 19-23] and references therein.

Here we extend the scope of such problems and consider the question of the largest cardinality of a set of points in the $n$-dimensional space over a finite field such that every triple of distinct points of this set defines an acute angle triangle. We note that a similar question in the Euclidean space $\mathcal{R}^{n}$ dates back to P. Erdôs and has been studied by several authors; see [1].

Certainly the notion of an acute triangle (or angle) is not immediately obvious in vector spaces over finite fields. Here we use the 'rational' interpretation of trigonometry invented by Wildberger [24] to extend this notion to finite fields.

To motivate our definition, we note that in the triangle defined by three distinct vectors $\mathbf{u}, \mathbf{v}, \mathbf{w} \in \mathbb{R}^{n}$, the vertex at $\mathbf{u}$ has an acute angle if and only if

$$
\|\mathbf{u}-\mathbf{v}\|^{2}+\|\mathbf{u}-\mathbf{w}\|^{2}-\|\mathbf{v}-\mathbf{w}\|^{2}>0,
$$

where $\|\mathbf{x}\|$ is the Euclidean norm of $\mathbf{x} \in \mathbb{R}^{n}$.

During the preparation of this paper, the author was supported in part by ARC grant DP0556431.

(C) 2009 Australian Mathematical Publishing Association Inc. 0004-9727/2009 \$16.00 
We now identify positive elements of a finite field $\mathbb{F}_{q}$ of $q$ elements with quadratic residues in $\mathbb{F}_{q}$ and say that, in the triangle defined by three distinct vectors

$$
\mathbf{u}=\left(u_{1}, \ldots, u_{n}\right), \quad \mathbf{v}=\left(v_{1}, \ldots, v_{n}\right), \quad \mathbf{w}=\left(w_{1}, \ldots, w_{n}\right) \in \mathbb{F}_{q}^{n},
$$

the vertex at $\mathbf{u}$ has an acute angle if any only if

$$
\Delta(\mathbf{u}, \mathbf{v}, \mathbf{w})=\sum_{i=1}^{n}\left(\left(u_{i}-v_{i}\right)^{2}+\left(u_{i}-w_{i}\right)^{2}-\left(v_{i}-w_{i}\right)^{2}\right)
$$

is a quadratic residue in $\mathbb{F}_{q}$.

Since in the field of even characteristic we always have $\Delta(\mathbf{u}, \mathbf{v}, \mathbf{w})=0$, this definition makes sense only if $q$ is odd.

We also remark that

$$
\begin{aligned}
\Delta(\mathbf{u}, \mathbf{v}, \mathbf{w}) & =2 \sum_{i=1}^{n}\left(u_{i}^{2}-u_{i} v_{i}-u_{i} w_{i}+v_{i} w_{i}\right) \\
& =2(\mathbf{u} \cdot \mathbf{u}-\mathbf{u} \cdot \mathbf{v}-\mathbf{u} \cdot \mathbf{w}+\mathbf{v} \cdot \mathbf{w})=2(\mathbf{u}-\mathbf{v}) \cdot(\mathbf{u}-\mathbf{w}),
\end{aligned}
$$

where $\mathbf{a} \cdot \mathbf{b}$ denotes the inner product of $\mathbf{a}, \mathbf{b} \in \mathbb{F}_{q}^{n}$. Thus, if $q$ is odd then $\Delta(\mathbf{u}, \mathbf{v}, \mathbf{w})=0$ if an only if $(\mathbf{u}-\mathbf{v}) \cdot(\mathbf{u}-\mathbf{w})=0$, which corresponds to orthogonality at $\mathbf{u}$ and thus to the Pythagoras theorem.

Let $N(n, q)$ be the largest possible cardinality of a set $\mathcal{Z} \subseteq \mathbb{F}_{q}$ such that all triples of distinct points $\mathbf{u}, \mathbf{v}, \mathbf{w} \in \mathcal{Z}$ define acute angle triangles.

We remark that $[8$, Theorem 1.1$]$ immediately yields

$$
N(n, q)=O\left(q^{(n+1) / 2}\right),
$$

where the implied constant depends only on $n$. In general, we do not know how to improve this bound. However, for $n=2$ we obtain a stronger estimate.

THEOREM 1. For a sufficiently large odd $q$,

$$
N(2, q) \leq 2 q^{4 / 3}
$$

\section{Additive character sums}

Let $\Psi$ be the set of all additive characters of $\mathbb{F}_{q}$ and let $\Psi^{*} \subset \Psi$ be the set of all nonprincipal characters; see [15, Section 11.1] for basic properties of additive characters. In particular, we also recall the identity

$$
\sum_{\psi \in \Psi} \psi(z)= \begin{cases}q & \text { if } z=0 \\ 0 & \text { otherwise }\end{cases}
$$

see [15, Section 11.1]. 
For an additive character $\psi \in \Psi$ and $\alpha \in \mathbb{F}_{q}$, we define the Gauss sum

$$
G_{\psi}(\alpha)=\sum_{z \in \mathbb{F}_{q}} \psi\left(\alpha z^{2}\right)=\sum_{z \in \mathbb{F}_{q}} \chi(z) \psi(\alpha z) \chi(\alpha) \sum_{z \in \mathbb{F}_{q}} \chi(z) \psi(z)=\chi(\alpha) G_{\psi}(1),
$$

where $\chi$ is the quadratic character in $\mathbb{F}_{q}$ (which exists since $q$ is odd), and recall that

$$
\left|G_{\psi}\right|=q^{1 / 2}
$$

for $\psi \in \Psi^{*}$ and $\alpha \in \mathbb{F}_{q}^{*}$; see [15, Proposition 11.5].

Finally, given a set $\mathcal{Z} \subseteq \mathbb{F}_{q}^{n}$, we define the triple character sum

$$
S_{\psi}(\mathcal{Z})=\sum_{\mathbf{u}, \mathbf{v}, \mathbf{w} \in \mathcal{Z}} \psi(\Delta(\mathbf{u}, \mathbf{v}, \mathbf{w}))
$$

Although we use our result on $S_{\psi}(\mathcal{Z})$ only in the case of $n=2$, here we present it in full generality as it may have some other applications.

LEMMA 2. For any $\psi \in \Psi^{*}$ and a set $\mathcal{Z} \subseteq \mathbb{F}_{q}^{n}$ of cardinality $Z=\# \mathcal{Z}$,

$$
\left|S_{\psi}(\mathcal{Z})\right|^{2} \leq Z q^{n} \sum_{\substack{\mathbf{v}, \mathbf{w}, \mathbf{x}, \mathbf{y} \in \mathcal{Z} \\ \mathbf{v}+\mathbf{w}=\mathbf{x}+\mathbf{y}}} \psi(2(\mathbf{v} \cdot \mathbf{w}-\mathbf{x} \cdot \mathbf{y}))
$$

PROOF. We have

$$
\left|S_{\psi}(\mathcal{Z})\right| \leq \sum_{\mathbf{u} \in \mathcal{Z}}\left|\sum_{\mathbf{v}, \mathbf{w} \in \mathcal{Z}} \psi(\Delta(\mathbf{u}, \mathbf{v}, \mathbf{w}))\right|
$$

Hence, recalling (1), we derive

$$
\left|S_{\psi}(\mathcal{Z})\right| \leq \sum_{\mathbf{u} \in \mathcal{Z}}\left|\sum_{\mathbf{v}, \mathbf{w} \in \mathcal{Z}} \psi(-2(\mathbf{u} \cdot(\mathbf{v}+\mathbf{w})-\mathbf{v} \cdot \mathbf{w}))\right| .
$$

Note that since $\psi(-z)=\overline{\psi(z)}$, we can replace -2 with 2 . By the Cauchy inequality

$$
\begin{aligned}
\left|S_{\psi}(\mathcal{Z})\right|^{2} & \leq Z \sum_{\mathbf{u} \in \mathcal{Z}}\left|\sum_{\mathbf{v}, \mathbf{w} \in \mathcal{Z}} \psi(2(\mathbf{u} \cdot(\mathbf{v}+\mathbf{w})-\mathbf{v} \cdot \mathbf{w}))\right|^{2} \\
& \leq Z \sum_{\mathbf{u} \in \mathbb{F}_{q}^{n}}\left|\sum_{\mathbf{v}, \mathbf{w} \in \mathcal{Z}} \psi(2(\mathbf{u} \cdot(\mathbf{v}+\mathbf{w})-\mathbf{v} \cdot \mathbf{w}))\right|^{2} \\
& =Z \sum_{\mathbf{u} \in \mathbb{F}_{q}^{n}} \sum_{\mathbf{v}, \mathbf{w}, \mathbf{x}, \mathbf{y} \in \mathcal{Z}} \psi(2(\mathbf{u} \cdot(\mathbf{v}+\mathbf{w}-\mathbf{x}-\mathbf{y})-\mathbf{v} \cdot \mathbf{w}+\mathbf{x} \cdot \mathbf{y})) \\
& =Z \sum_{\mathbf{v}, \mathbf{w}, \mathbf{x}, \mathbf{y} \in \mathcal{Z}} \psi(2(\mathbf{x} \cdot \mathbf{y}-\mathbf{v} \cdot \mathbf{w})) \sum_{\mathbf{u} \in \mathbb{F}_{q}^{n}} \psi(2 \mathbf{u} \cdot(\mathbf{v}+\mathbf{w}-\mathbf{x}-\mathbf{y})) .
\end{aligned}
$$


Finally, changing the order of summation, and replacing $2 \mathbf{u}$ with $\mathbf{u}$, we obtain

$$
\left|S_{\psi}(\mathcal{Z})\right|^{2} \leq Z \sum_{\mathbf{v}, \mathbf{w}, \mathbf{x}, \mathbf{y} \in \mathcal{Z}} \psi(2(\mathbf{x} \cdot \mathbf{y}-\mathbf{v} \cdot \mathbf{w})) \sum_{\mathbf{u} \in \mathbb{F}_{q}^{n}} \psi(\mathbf{u} \cdot(\mathbf{v}+\mathbf{w}-\mathbf{x}-\mathbf{y})) .
$$

By the orthogonality property of additive characters (3), we see that the inner sum vanishes if and only if

$$
\mathbf{v}+\mathbf{w}-\mathbf{x}-\mathbf{y}=0
$$

in which case it equals $q^{n}$. Now renaming the variables $(\mathbf{v}, \mathbf{w}) \leftrightarrow(\mathbf{x}, \mathbf{y})$, we conclude the proof.

\section{Proof of Theorem 1}

Assume that for the set $\mathcal{Z} \subseteq \mathbb{F}_{q}^{2}$ all triples of distinct vectors $\mathbf{u}, \mathbf{v}, \mathbf{w} \in \mathcal{Z}$ define acute angle triangles. As before, use $Z=\# \mathcal{Z}$ to denote the cardinality of $\mathcal{Z}$. Fix an arbitrary quadratic nonresidue $\alpha \in \mathbb{F}_{q}$. Then we see that the equation

$$
\Delta(\mathbf{u}, \mathbf{v}, \mathbf{w})=\alpha z^{2}, \quad \mathbf{u}, \mathbf{v}, \mathbf{w} \in \mathcal{Z}, \quad z \in \mathbb{F}_{q},
$$

has at most

$$
T \leq Z^{2}
$$

solutions (which come only from the triples $\mathbf{u}, \mathbf{v}, \mathbf{w} \in \mathcal{Z}$ with $\Delta(\mathbf{u}, \mathbf{v}, \mathbf{w})=0$, that is, when $\mathbf{u}=\mathbf{v}$ or $\mathbf{u}=\mathbf{w})$.

On the other hand, from the orthogonality property of characters (3), we obtain

$$
T=\sum_{\mathbf{u}, \mathbf{v}, \mathbf{w} \in \mathcal{Z}} \sum_{z \in \mathbb{F}_{q}} \frac{1}{q} \sum_{\psi \in \Psi} \psi\left(\Delta(\mathbf{u}, \mathbf{v}, \mathbf{w})-\alpha z^{2}\right)=\frac{1}{q} \sum_{\psi \in \Psi} G_{\psi}(-\alpha) S_{\psi}(\mathcal{Z}) .
$$

The term corresponding to the principal character $\psi=\psi_{0}$ is equal to $Z^{3}$. Thus, recalling (4), we obtain

$$
\left|T-Z^{3}\right| \leq q^{-1 / 2} R
$$

where

$$
R=\sum_{\psi \in \Psi^{*}}\left|S_{\psi}(\mathcal{Z})\right|
$$

Now, by the Cauchy inequality,

$$
R^{2} \leq q \sum_{\psi \in \Psi^{*}}\left|S_{\psi}(\mathcal{Z})\right|^{2}
$$

Thus using Lemma 2 and then extending the summation to all $\psi \in \Psi$, we deduce that

$$
R^{2} \leq Z q^{3} \sum_{\psi \in \Psi} \sum_{\substack{\mathbf{v}, \mathbf{w}, \mathbf{x}, \mathbf{y} \in \mathcal{Z} \\ \mathbf{v}+\mathbf{w}=\mathbf{x}+\mathbf{y}}} \psi(2(\mathbf{v} \cdot \mathbf{w}-\mathbf{x} \cdot \mathbf{y}))
$$


Changing the order of summation and using (3) again, we obtain

$$
R^{2} \leq Z q^{3} \sum_{\substack{\mathbf{v}, \mathbf{w}, \mathbf{x}, \mathbf{y} \in \mathcal{Z} \\ \mathbf{v}+\mathbf{w}=\mathbf{x}+\mathbf{y}}} \sum_{\psi \in \Psi} \psi(2(\mathbf{v} \cdot \mathbf{w}-\mathbf{x} \cdot \mathbf{y}))=Z q^{4} W
$$

where $W$ is the number of solutions to the system of equations

$$
\mathbf{v}+\mathbf{w}=\mathbf{x}+\mathbf{y} \quad \text { and } \quad \mathbf{v} \cdot \mathbf{w}=\mathbf{x} \cdot \mathbf{y}
$$

in $\mathbf{v}, \mathbf{w}, \mathbf{x}, \mathbf{y} \in \mathcal{Z}$, which is the same as the number of solutions to the equation

$$
\mathbf{v} \cdot \mathbf{w}=\mathbf{x} \cdot(\mathbf{v}+\mathbf{w}-\mathbf{x})
$$

in $\mathbf{v}, \mathbf{w}, \mathbf{x} \in \mathcal{Z}$. Clearly, when $\mathbf{v}, \mathbf{w}$ and one component of $\mathbf{x}$ are fixed, we obtain a nontrivial quadratic equation over $\mathbb{F}_{q}$ for the other component of $\mathbf{x}$. Therefore

$$
W \leq 2 Z^{2} q
$$

Substituting in (7), we obtain

$$
R^{2} \leq 2 Z^{3} q^{5}
$$

In turn, inserting this estimate in (6) yields

$$
\left|T-Z^{3}\right| \leq \sqrt{2} Z^{3 / 2} q^{2}
$$

If $Z<2 q^{4 / 3}$ then there is nothing to prove. Otherwise,

$$
\sqrt{2} Z^{3 / 2} q^{2} \leq \frac{1}{2} Z^{3}
$$

thus, by (8) we obtain

$$
T \geq \frac{1}{2} Z^{3}
$$

which contradicts (5), provided that $q$ is large enough.

\section{Remarks}

Unfortunately the method of this paper, although it works for any $n$, leads to a bound which is the same as (2) for $n=3$ and is even weaker than (2) for $n \geq 4$.

Furthermore, using the bound

$$
\left|S_{\psi}(\mathcal{Z})\right| \leq Z^{2} q^{n / 2}
$$

(which is immediate from Lemma 2) in the argument of the proof of Theorem 1, one can recover the bound (2), but it does not seem to give anything stronger that this.

An alternative way to estimate $N(n, q)$ is via bounds of quadratic character sums

$$
T_{\chi}(\mathcal{Z})=\sum_{\mathbf{u}, \mathbf{v}, \mathbf{w} \in \mathcal{Z}} \chi(\Delta(\mathbf{u}, \mathbf{v}, \mathbf{w})) .
$$


Using the same approach (via the Cauchy inequality and extending summation over $\mathbf{u} \in \mathcal{Z}$ to the full space $\mathbb{F}_{q}^{n}$ ) as in the proof of Lemma 2, we obtain

$$
\left|T_{\chi}(\mathcal{Z})\right|^{2}=Z \sum_{\mathbf{v}, \mathbf{w}, \mathbf{x}, \mathbf{y} \in \mathcal{Z}} \sum_{\mathbf{u} \in \mathbb{F}_{q}^{n}} \chi(\Delta(\mathbf{u}, \mathbf{v}, \mathbf{w}) \Delta(\mathbf{u}, \mathbf{x}, \mathbf{y})) .
$$

It is natural to conjecture that the inner sum admits a square root estimate and thus is $O\left(q^{n / 2}\right)$ unless $(\mathbf{v}, \mathbf{w})$ is a permutation of $(\mathbf{x}, \mathbf{y})$. One can derive that $N(n, q)=$ $O\left(q^{n / 2}\right)$ from such a hypothetical bound. Unfortunately the highest form of the polynomial

$$
F_{\mathbf{v}, \mathbf{w}, \mathbf{x}, \mathbf{y}}(\mathbf{U})=\Delta(\mathbf{U}, \mathbf{v}, \mathbf{w}) \Delta(\mathbf{U}, \mathbf{x}, \mathbf{y}) \in \mathbb{F}[\mathbf{U}]
$$

is singular, so known analogues of the Deligne bound for multivariate character sums (see $[16,18])$ do not apply.

We recall that in $\mathbb{R}^{n}$, the largest number of vectors such that each triple defines an acute angle triangle is bounded by a function of $n$; see [1]. Although our bounds seem to be much higher that the true order of magnitude of $N(n, q)$, we observe that $\lim \sup _{p \rightarrow \infty} N(n, p)=\infty$. Indeed, by the result of Graham and Ringrose [7], there is an absolute constant $C>0$ such that, for infinitely many primes $p$, all nonnegative integers $z \leq C \log p \log \log \log p$ are quadratic residues modulo $p$. Thus, for each such $p$ and an appropriate constant $c$, for the set $\mathcal{Z} \subseteq \mathbb{F}_{p}$ formed by vectors with components in the interval $[1, c \sqrt{\log p \log \log \log p}]$ we have

$$
1 \leq \Delta(\mathbf{u}, \mathbf{v}, \mathbf{w}) \leq C \log p \log \log \log p
$$

for any pairwise distinct $\mathbf{u}, \mathbf{v}, \mathbf{w} \in \mathcal{Z}$ and thus $\Delta(\mathbf{u}, \mathbf{v}, \mathbf{w})$ is a quadratic residue. This implies that

$$
\limsup _{p \rightarrow \infty} \frac{N(n, p)}{(\log p \log \log \log p)^{n / 2}}>0 .
$$

\section{Acknowledgements}

The author is grateful to Norman Wildberger for his suggestion of an $\mathbb{F}_{q}$-interpretation of acute angle triangles, which has been used here. The author would also like to thank Nick Katz for a useful discussion of some issues related to possible estimates of the quadratic character sums $T_{\chi}(\mathcal{Z})$.

\section{References}

[1] E. Ackerman and O. Ben-Zwi, 'On sets of points that determine only acute angles', European J. Combin. 30 (2009), 908-910.

[2] J. Bourgain and M. Z. Garaev, 'On a variant of sum-product estimates and explicit exponential sum bounds in prime fields', Math. Proc. Cambridge Philos. Soc. 146 (2008), 1-21.

[3] J. Bourgain, N. Katz and T. Tao, 'A sum product estimate in finite fields and applications', Geom. Funct. Anal. 14 (2004), 27-57.

[4] D. Covert, D. Hart, A. Iosevich, D. Koh and M. Rudnev, 'Generalized incidence theorems, homogeneous forms and sum-product estimates in finite fields', European J. Combin., to appear. 
[5] Z. Dvir, 'On the size of Kakeya sets in finite fields', J. Amer. Math. Soc. 22 (2009), 1093-1097.

[6] M. Z. Garaev, 'The sum-product estimate for large subsets of prime fields', Proc. Amer. Math. Soc. 136 (2008), 2735-2739.

[7] S. W. Graham and C. J. Ringrose, 'Lower bounds for least quadratic nonresidues', in: Analytic Number Theory, Allerton Park 1989, Progress in Mathematics, 85 (Birkhäuser, Basel, 1990), pp. 269-309.

[8] D. Hart and A. Iosevich, 'Ubiquity of simplices in subsets of vector spaces over finite fields', Anal. Math. 34 (2007), 29-38.

[9] D. Hart, A. Iosevich, D. Koh and M. Rudnev, 'Averages over hyperplanes, sum-product theory in finite fields', Trans. Amer. Math. Soc., to appear.

[10] D. Hart, A. Iosevich and J. Solymosi, 'Sums and products in finite fields via Kloosterman sums', Int. Math. Res. Not. 2007 (2007), 1-14; Article ID rnm007.

[11] A. Iosevich and D. Koh, 'Erdős-Falconer distance problem, exponential sums, and Fourier analytic approach to incidence theorems in vector spaces over finite fields', SIAM J. Discrete Math. 23 (2008), 123-135.

[12] A. Iosevich and M. Rudnev, 'Erdős distance problem in vector spaces over finite fields', Trans. Amer. Math. Soc. 359 (2007), 6127-6142.

[13] A. Iosevich and S. Senger, 'Orthogonal systems in vector spaces over finite fields', Electron. J. Combin. 15 (2008), Article R151.

[14] A. Iosevich, I. E. Shparlinski and M. Xiong, 'Sets with integral distances in finite fields', Trans. Amer. Math. Soc., to appear.

[15] H. Iwaniec and E. Kowalski, Analytic Number Theory (American Mathematical Society, Providence, RI, 2004).

[16] N. M. Katz, 'Estimates for nonsingular multiplicative character sums', Int. Math. Res. Not. 2002 (2002), 333-349.

[17] N. H. Katz and C.-Y. Shen, 'Garaev's inequality in finite fields not of prime order', J. Anal. Combin. 3 (2008), Article 3.

[18] G. I. Perel'muter, 'Estimation of a multiple sum with the Legendre symbol', Mat. Zametki 18 (1975), 421-427 (in Russian).

[19] C.-Y. Shen, 'An extension of Bourgain and Garaev's sum-product estimates', Acta Arith. 135 (2008), 351-356.

[20] L. A. Vinh, 'Explicit Ramsey graphs and Erdôs distance problem over finite Euclidean and nonEuclidean spaces', Electron. J. Combin. 15 (2008), Aricle R5.

[21] L. A. Vinh, 'On the number of orthogonal systems in vector spaces over finite fields', Electron. J. Combin. 15 (2008), Aricle N32.

[22] L. A. Vinh, 'On a Furstenberg-Katznelson-Weiss type theorem over finite fields', Ann. Combin., to appear.

[23] L. A. Vinh, 'Szemeredi-Trotter type theorem and sum-product estimate in finite fields', European J. Combin., to appear.

[24] N. J. Wildberger, Divine Proportions: Rational Trigonometry to Universal Geometry (Wild Egg Books, Sydney, 2005).

IGOR E. SHPARLINSKI, Department of Computing, Macquarie University, Sydney, NSW 2109, Australia

e-mail: igor@ics.mq.edu.au 